\title{
IRIS IMAGE KEY POINTS DESCRIPTORS BASED ON PHASE CONGRUENCY
}

\author{
M. A. Protsenko, E. A. Pavelyeva \\ Faculty of Computational Mathematics and Cybernetics, Lomonosov Moscow State University, \\ 119991, Russia, Moscow, Leninskie Gory, MGU \\ ma.protsenko@mail.ru, paveljeva@yandex.ru
}

Commission II, WG II/10

KEY WORDS: Biometrics, iris recognition, key points, phase, phase congruency

\begin{abstract}
:
In this article the new method for iris image features extraction based on phase congruency is proposed. Iris image key points are calculated using the convolutions with Hermite transform functions. At each key point the feature vector characterizing this key point is obtained based on the phase congruency method. Iris key point descriptor contains phase congruency values at points located on concentric circles around the key point. To compare the key points, Euclidean metric between the key points descriptors is calculated. The distance between the iris images is equal to the number of matched iris key points. The proposed method was tested using the images from CASIA-IrisV4-Interval database and the value of EER $=0.226 \%$ was obtained.
\end{abstract}

\section{INTRODUCTION}

Iris recognition is one of the most powerful biometric technologies for human identification. Iris is used in different fields of access control and security due to its high reliability, accuracy and uniqueness. Traditional iris recognition systems use near-infrared sensors to acquire images of the iris but this restricts the iris image acquisition distance. Over the last several years, there have been numerous attempts to design and implement iris recognition systems that operate at longer distances but that yields some problem both in hardware and algorithmic perspectives. (Nguyen, 2017). Mobile biometrics represent now the new trends of authentication. The most appealing feature of mobile devices is the wide availability and the presence of reliable sensors for capturing biometric traits, e.g., cameras and accelerometers. Moreover, they more and more often store personal and sensitive data, that need to be protected. Doing this on the same device using biometrics to enforce security seems a natural solution. Thus the use of iris recognition for mobile devices is generally promising (De Marsico, 2017). Some methods use also both the iris and periocular information (Santos, 2015) to obtain more robust results.

There are three main stages in iris recognition system: image preprocessing, feature extraction and feature matching. Different approaches for solution of iris recognition problem mostly differ in iris feature extraction and matching algorithms, although machine learning techniques are also widely used. (De Marsico, 2016, Ahuja, 2016). The first and the most famous iris recognition method was proposed by J. Daugman (Daugman, 2004). In Daugman's method 2-D Gabor wavelets are used to obtain the phase information of iris image. The phase is then encoded and the 256-byte "iris code" is obtained. Later it was shown (Hollingsworth, 2009) that some iris regions contain more information than others. Iris texture is also unstable to strong illumination changes where the non-linear distortions of iris features may occur. So the feature extraction methods based on iris key points look promising to be used in iris recognition (Alvarez-Betancourt, 2016, Sahu, 2018). The iris key points matching methods can use the information about iris texture in local areas of key points to obtain more robust results.

The key points can be extracted by different algorithms. In particular, numerous SIFT-based (Lowe, 1999) schemes are presented (Rathgeb, 2018). SIFT descriptor can also be used to describe distinctive features for different types of key points (Alvarez-Betancourt, 2016). After key points extraction the density-based spatial clustering and key point reduction can be applied to reduce the time for iris recognition (Sahu, 2018).

In this article we find the iris key points using Hermite transform functions and propose a new method for iris image features extraction based on phase congruency. Phase congruency can measure the significance of image features and it provides a measure that is independent of the overall magnitude of the signal. Phase congruency can be used for biometric image enhancement (Punsawad, 2009), for edge and corner detection (Kovesi, 2003) and in many other problems of image processing (Pavelyeva, 2018).

The rest of this paper is arranged as follows: in Section 2 the iris key points extraction algorithm is described. The phase congruency method is discussed in Section 3, and it is shown how the phase congruency can measure the significance of image features. In section 4 the method of iris key point descriptors calculation is presented. The experimental results are shown in Section 5. Finally, Section 6 provides some conclusions.

\section{IRIS IMAGE PREPROCESSING AND KEY POINTS EXTRACTION}

In this research the iris images from CASIA-IrisV4-Interval database (CASIA, 2010) are used (Fig. 1). After iris localization the iris is normalized to a rectangular image and iris areas free of eyelashes, eyelids and glares are determined. The normalized iris image can have low contrast and non-uniform brightness caused by the position of light sources so the image enhancement algorithm is applied (Fig. 2).

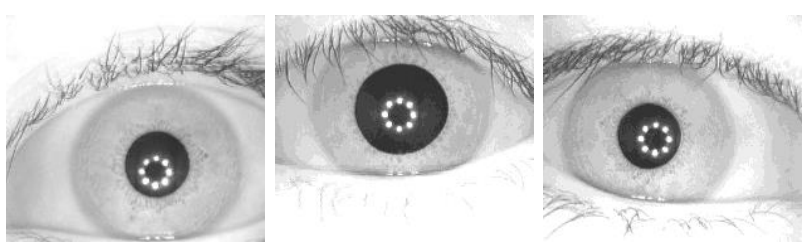

Figure 1. Examples of iris images from CASIA-IrisV4-Interval database 


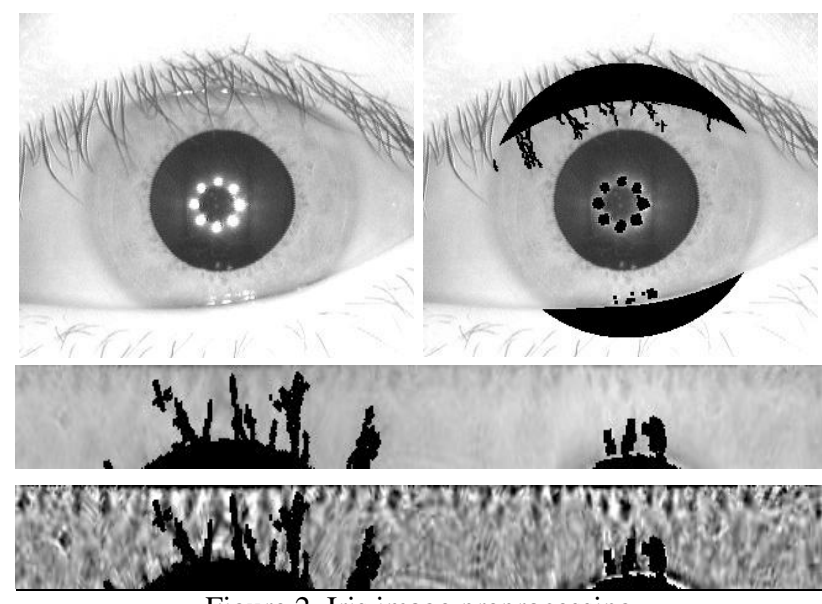

Figure 2. Iris image preprocessing

Then the key points are calculated using the convolutions of normalized iris image with the Hermite transform functions

$$
\varphi_{m, n}(x, y)=\frac{(-1)^{m+n} \cdot e^{-\left(\frac{x^{2}}{\left.\sigma_{x}^{2}+\frac{y^{2}}{\sigma_{y}^{2}}\right)}\right.}}{\sqrt{2^{m+n} \cdot m ! \cdot n !} \cdot \pi} \cdot \frac{1}{\sigma_{x} \sigma_{y}} \cdot H_{n}\left(\frac{x}{\sigma_{x}}\right) H_{m}\left(\frac{y}{\sigma_{y}}\right),
$$

where $H_{n}(x)$ are the Hermite polynomials:

$$
\begin{aligned}
& H_{0}(x)=1, \quad H_{1}(x)=2 \cdot x, \\
& H_{n}(x)=2 \cdot x \cdot H_{n-1}(x)-2 \cdot(n-1) \cdot H_{n-2}(x), n \geq 2 .
\end{aligned}
$$

We convolve the normalized image with $\varphi_{2,0}(x, y)$, $\sigma_{x}=\sigma_{y}=3$ :

$$
M_{2,0}(x, y)=I(x, y) * \varphi_{2.0}(x, y) .
$$

Then we consider the points where the convolution values are more than one third of $\max _{(x, y)}\left(M_{2,0}(x, y)\right)$ with an additional assumption that the distance between the points shall be not less than 4 pixels (Fig. 3). We call the taken points as iris key points (Pavelyeva, 2013) and suppose that iris image can have no more than 75 key points. At each key point the feature vector characterizing this key point is calculated based on the phase congruency method.
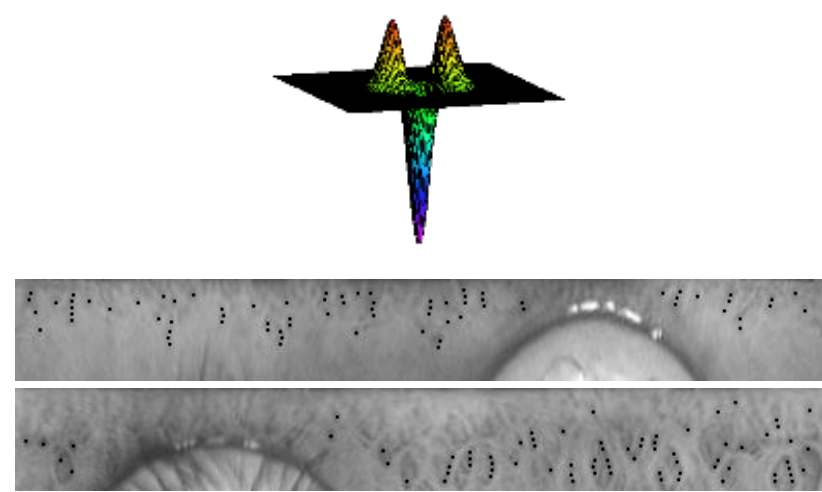

Figure 3. $\varphi_{2,0}(x, y)$ and the examples of iris key points extraction

\section{PHASE CONGRUENCY}

The one dimensional wavelet transform at point $p$ and scale $s$ for the signal $f(x)$ is defined as

$$
F(s, p)=\left.\left[f(x) * \frac{1}{\sqrt{s}} G\left(\frac{x}{s}\right)\right]\right|_{x=p},
$$

where $G(x)$ is the wavelet function, $s \in R^{+}$is the scale parameter, $p \in R$ is the shift parameter, $*$ denotes signal convolution. The wavelet transform rewrites the signal to the time-frequency representation.

We denote the values of the wavelet transform magnitude and phase as $A_{s}(p)$ and $\varphi_{s}(p) ; \quad F(p)=\sum_{s} \operatorname{Re} F(s, p)$, $H(p)=\sum_{s} \operatorname{Im} F(s, p), \quad E(p)=\sqrt{F^{2}(p)+H^{2}(p)}$. It can be shown that the values of $\varphi_{s}(p)$ have a small difference over all scales $s$ at feature points of the signal (Kovesi, 2003). In Fig. 4 and Fig. 5 a one dimensional signal and its phase scalogram (the values of $\varphi_{s}(p)$ for different scales $s$ ) are presented (Kovesi, 1996). If a signal has step edge, then we can observe a vertical line of constant grey value in the scalogram that means that the phase of wavelet transform does not change significantly over all scales at given point. In Fig. 4 and Fig. 5 the phase is mapped from 0-360 degrees to 0-255 gray levels for visualization.

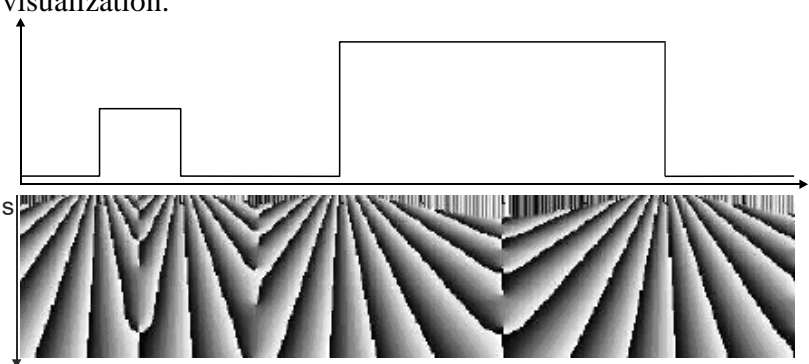

Figure 4. A one dimensional signal and its phase scalogram

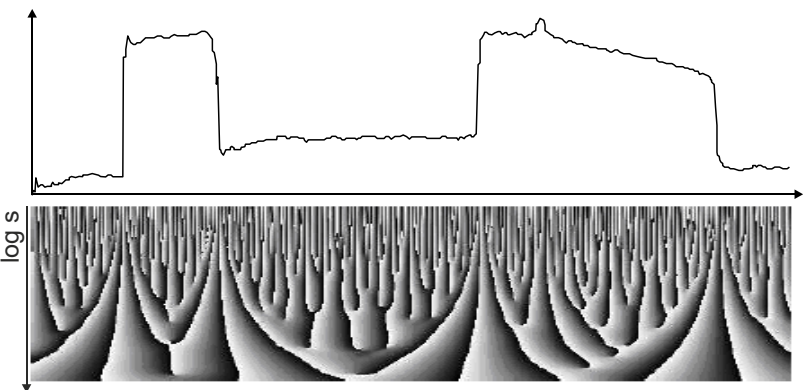

Figure 5. A one dimensional noisy signal and its phase scalogram

The phase congruency at point $p$ is defined as

$$
P C(p)=\frac{E(p)}{\sum_{s} A_{s}(p)}=\frac{\left|\sum_{s} F(s, p)\right|}{\sum_{s} A_{s}(p)} .
$$

It can be seen that $P C(p) \in[0,1]$. A geometric interpretation of phase congruency is shown in Fig 6. For a fixed point $p$ each convolution $F(s, p)$ can be represented as a vector with the length equal to the magnitude $A_{s}(p)$, and the angle equal to $\varphi_{s}(p)$. If there is a feature at a given point $p$ of the image, the value of $P C(p)$ is close to 1 , because at this point the phase values $\varphi_{s}(p)$ will have a small difference at all scales $s$, and vectors $F(s, p)$ will be practically collinear. Thus the phase 
congruency can measure the significance of image features. Phase congruency provides a measure that is independent of the overall magnitude of the signal making it invariant to variations in image illumination and contrast (Kovesi, 2003).

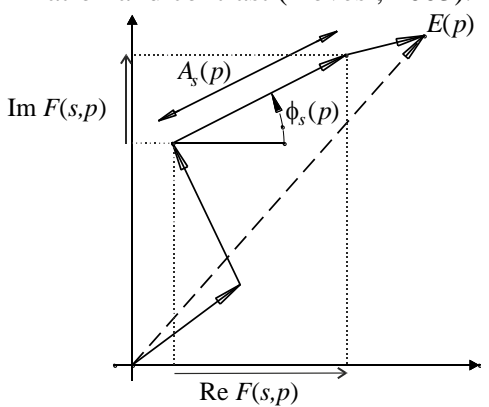

Figure 6. A geometric interpretation of phase congruency

We use 2D Log-Gabor filters to calculate phase congruency. The Log-Gabor functions have Gaussian transfer functions at the logarithmic frequency scale while Gabor functions have Gaussian transfer functions at the linear frequency scale. Unlike Gabor filters, the Log-Gabor filters have zero DC component and there are no limitations in the maximum bandwidth that can be obtained from Log-Gabor functions (Fig. 7). The Log-Gabor functions are defined in the frequency domain (Field, 1987):

$$
G(f, \theta)=\exp \left(\frac{-\left(\log \left(\omega / \omega_{0}\right)\right)^{2}}{2\left(\log \left(\sigma_{\omega} / \omega_{0}\right)\right)}\right) \exp \left(\frac{-\left(\theta-\theta_{0}\right)^{2}}{2 \sigma_{\theta}^{2}}\right),
$$

where $\omega_{0}$ is the filter's centre frequency, $\theta_{0}$ represents the orientation of the filter, $\sigma_{\omega}$ and $\sigma_{\theta}$ are the width parameters. To obtain constant shape ratio filters the term $\sigma_{\omega} / \omega_{0}$ should be constant for varying $\omega$. For example, a $\sigma_{\omega} / \omega_{0}$ value of 0.74 will result in a filter bandwidth of approximately one octave, 0.55 will result in two octaves, and 0.41 will produce three octaves (Xiao, 2004). The orientation multiplier is a Gaussian distance function according to the angle in polar coordinate. (Field, 1987). An analytic expression for the shape of the LogGabor functions cannot be constructed in the spatial domain due to the singularity in the $\log$ function at the origin (Kovesi, 1996). Log-Gabor filters can be constructed with arbitrary bandwidth and the bandwidth can be optimized to produce a filter with minimal spatial extent.

\section{Gabor}

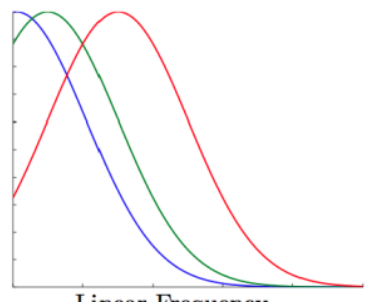

Linear Frequency

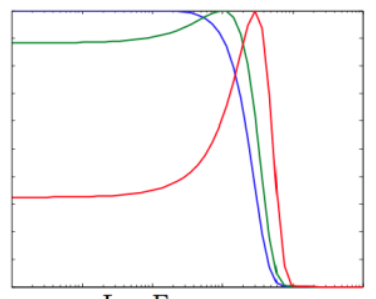

Log Frequency

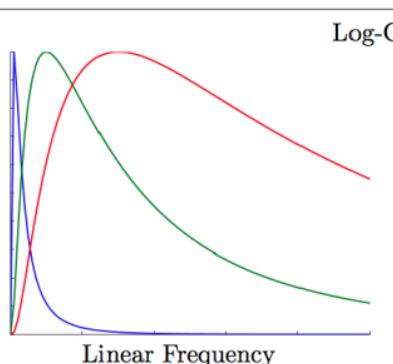

Linear Frequency

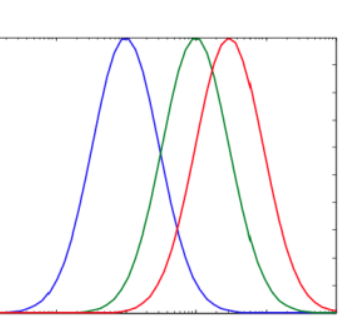

Log Frequency

Figure 7. Gabor and log Gabor functions viewed on linear and logarithmic frequency scales
In two-dimensional case the phase congruency at point $(p, q)$ is defined as

$$
P C(p, q)=\frac{\sum_{\theta}\left|\sum_{s} F(s, p, q)\right|}{\sum_{\theta} \sum_{s}|F(s, p, q)|},
$$

where the two-dimensional wavelet transform $F(s, p, q)$ for an image with Log-Gabor function is calculated using the convolution theorem. The example of normalized iris image and its phase congruency map $F(s, p, q)$ is presented in Fig. 8. The phase congruency values are mapped from [0,1] to 0-255 gray levels for visualization. Here 4 scales and 6 orientations are used and the parameters $\omega_{0}=0.1, \sigma_{\omega}=0.07, \sigma_{\theta}=0.4$ are taken.

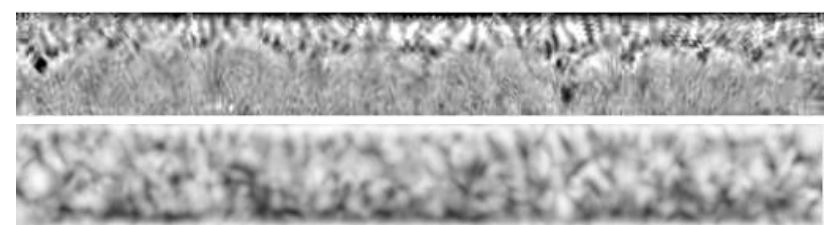

Figure 8 . The normalized iris image and its phase congruency map

\section{IRIS KEY POINTS DESCRIPTORS}

At each iris key point feature vector contains phase congruency values at 24 points located on two concentric circles (radius $r$ and $2 r$ ) around the key point, where $r=\sigma_{x}, \sigma_{x}$ was used in iris key points extraction stage. This feature vector we call as iris key point descriptor. If $(x, y)$ are the key point coordinates then the points $\left(x_{i}, y_{i}\right), i=1,2, \ldots, 24$ have the following coordinates:

$$
\begin{aligned}
& x_{i}=x+r \cos \left(\frac{j \pi}{4}\right), y_{i}=y+r \sin \left(\frac{j \pi}{4}\right), j=0 . .7, i=j+1, \\
& x_{i}=x+2 r \cos \left(\frac{j \pi}{8}\right), y_{i}=y+2 r \sin \left(\frac{j \pi}{8}\right), j=0 . .15, i=j+9 .
\end{aligned}
$$

The taken points are shown in Fig. 9. For better visualization, in Fig. 10. only part of the image and only 8 key points are presented.

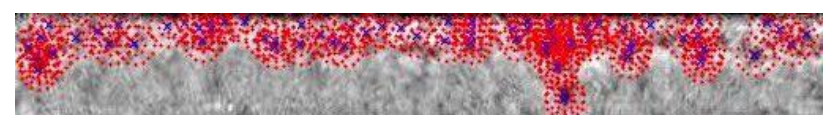

Figure 9. Iris image key points (blue) and points around them (red) used to calculate key points descriptors

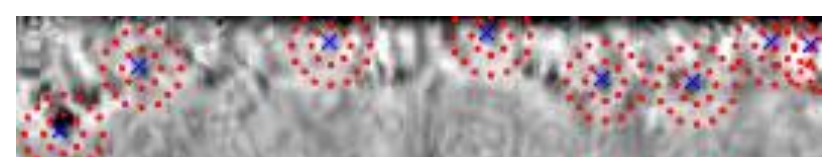

Figure 10. Some iris image key points (blue crosses) and points around them used to calculate key points descriptors

To compare the key points, Euclidean metric between the feature vectors is calculated. Points outside the image have zero values, and these points are not used in Euclidean metric. To obtain the final result we divide the derived distance to the number of the used values. Thus the distance between two comparable key points $P_{1}$ and $P_{2}$ with feature vectors $a=\left(a_{1}, \ldots, a_{24}\right)$ and $b=\left(b_{1}, \ldots, b_{24}\right)$ is 


$$
\operatorname{dist}\left(P_{1}, P_{2}\right)=\frac{\sqrt{\sum_{i \in I}\left(a_{i}-b_{i}\right)^{2}}}{|I|},
$$

where $I=\left\{i \in\{1.24\} \mid a_{i} \cdot b_{i} \neq 0\right\},|I|$ is the cardinality of $I$.

If the distance between the key points of two iris images is less than a given threshold, then these key points are considered as belonging to the same iris texture area. We assume that the spatial shift between the comparable key points may correspond to the angle of eye rotation no more than 30 degrees.

\section{EXPERIMENTAL RESULTS}

The proposed method was tested with a part of CASIA-IrisV4-Interval database. In our research 6 orientations and 4 scales of Log-Gabor functions were taken. The examples of iris key points matching for two images of one eye are shown in Fig. 11. The red lines connect the matching key points. The example of iris key points matching for two images of different eyes is shown in Fig. 12.
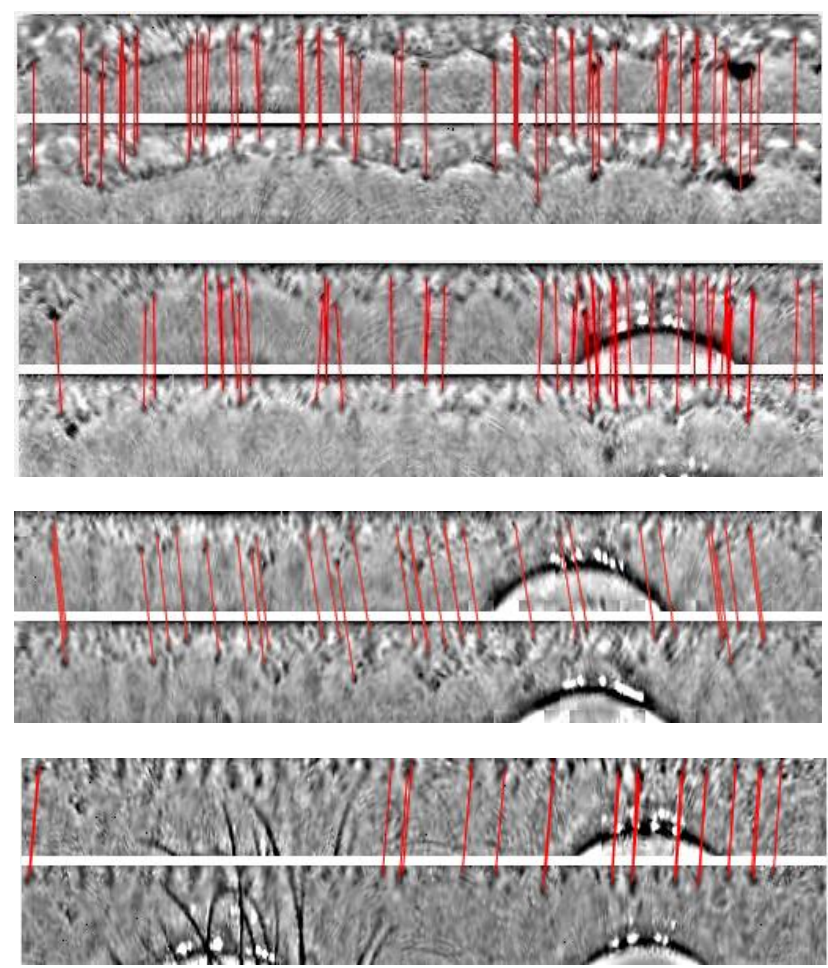

Figure 11. The examples of key points matching for two iris images of one eye. The red lines connect the matching key points

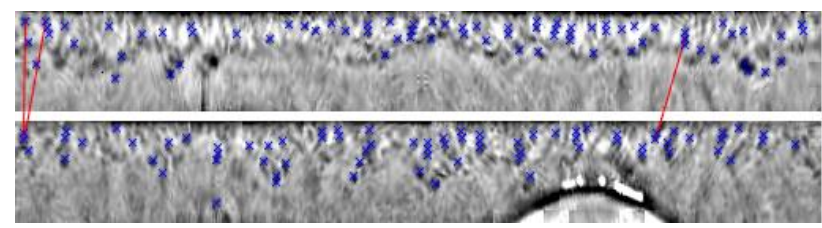

Figure 12. The example of key points extraction and key points matching for two iris images of different eyes: 3 matched key points are detected

The distance between the iris images is equal to the number of matched iris key points. The distribution of genuine and impostor scores is demonstrated in Fig. 13. The ROC-curve is shown in Fig. 14. The value of EER $=0.226 \%$ was obtained.

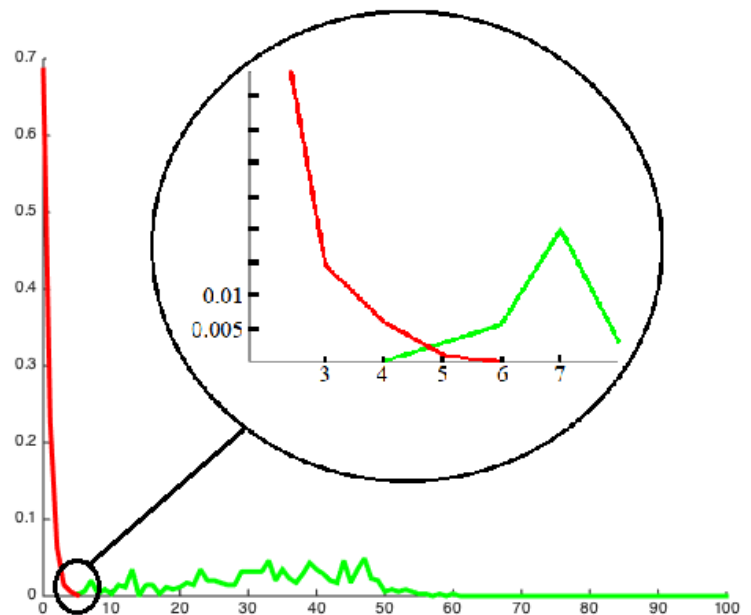

Figure 13. The distribution of genuine (green line) and impostor (red line) scores

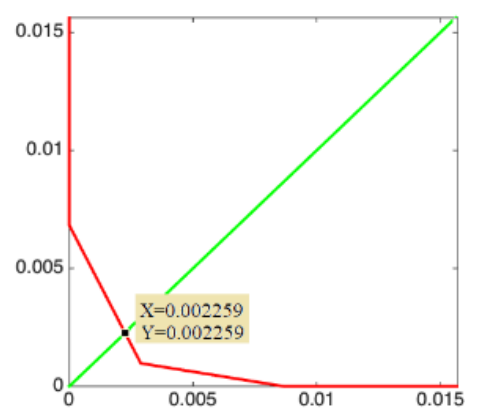

Figure 14. ROC-curve, $\mathrm{EER}=0.226 \%$

\section{CONCLUSIONS}

The new method for iris key points descriptors calculation is described in the article. The proposed method was tested using the images from CASIA-IrisV4-Interval database and the value of EER $=0.226 \%$ was obtained. The key points are calculated based on Hermite transform. The key point descriptor consists of phase congruency values at points around taken key point. The proposed method of iris key points descriptors calculation using phase congruency looks promising to be used in iris recognition.

\section{REFERENCES}

Ahuja K., Islam R., Barbhuiya F. A., Dey K., 2016. A preliminary study of CNNs for iris and periocular verification in the visible spectrum. ICPR, pp. 181-186.

Alvarez-Betancourt Y. and Garcia-Silvente M., 2016. A keypoints-based feature extraction method for iris recognition under variable image quality conditions. Knowledge-Based Systems, 92, pp. 169-182.

CASIA-IrisV4-Interval database. 2010.

http://www.cbsr.ia.ac.cn/english/IrisDatabase.asp

Daugman J., 2004. How iris recognition works. IEEE Transactions on Circuits and Systems for Video Technology, 14(1), pp. 21-30.

De Marsico M., Petrosino A., Ricciardi S., 2016. Iris recognition through machine learning techniques: A survey. Pattern Recognition Letters. 82, pp. 106-115. 
De Marsico M., Nappi M., Proença H., 2017. Results from miche ii-mobile iris challenge evaluation ii. Pattern Recognition Letters, 91, pp. 3-10.

Field D. J., 1987. Relations between the statistics of natural images and the response properties of cortical cells. Josa A, 4(12), pp. 2379-2394.

Hollingsworth K. P., Bowyer K. W., and Flynn P. J., 2009. The best bits in an iris code. IEEE Transactions on Pattern Analysis and Machine Intelligence. 31(6), pp. 964-973.

Kovesi P., 1996. Invariant measures of image features from phase information, $\mathrm{PhD}$ thesis, The University of Western Australia.

Kovesi P., 2003. Phase congruency detects corners and edges. Proceedings of the Digital Image Computing: Techniques and Applications (DICTA), pp. 309-318.

Lowe D. G. 1999. Object recognition from local scale-invariant features. ICCV, 99(2), pp. 1150-1157.

Nguyen K., Fookes C., Jillela R., Sridharan S., Ross A., 2017. Long range iris recognition: A survey. Pattern Recognition, 72, pp. 123-143.

Pavelyeva E. A., 2013. The search for matches between the iris key points using Hermite projection phase-only correlation method. Systems and Means of Informatics, 23(2), pp. 74-88 [In Russian].

Pavelyeva, E. A., 2018. Image processing and analysis based on the use of phase information. Computer Optics, 42(6), pp. 10221034 .

Punsawad Y., Wongsawat Y., 2008. Palmprint image enhancement using phase congruency. Robotics and Biomimetics (ROBIO), pp. 1643-1646.

Rathgeb C., Wagner J., Busch C., 2018. SIFT-based iris recognition revisited: prerequisites, advantages and improvements. Pattern Analysis and Applications, pp. 1-18.

Sahu B., Sa P. K., Bakshi S., and Sangaiah A. K., 2018. Reducing dense local feature key-points for faster iris recognition. Computers and Electrical Engineering, 70, 939949.

Santos G., Grancho E., Bernardo M. V., Fiadeiro, P. T., 2015. Fusing iris and periocular information for cross-sensor recognition. Pattern Recognition Letters, 57, pp. 52-59.

Xiao Z., Hou Z., Guo Y., 2004. Log Gabor wavelet — consistent with human visual system characteristics. Wavelet Analysis and Its Applications, and Active Media Technology, 1, pp. 45-50. 